seismic energy is dissipated by a profound crustal discontinuity at the edge of the continental shelf. Hurricanes crossing the edge suddenly gonerate larger microseisms".

Observations are still being made at observatories throughout the world, in conformity with the microseismic research project of the Section of Seismology of the International Geodetic and Geophysical Union, which was initiated in 1952, and detailed observations have yet to be made of the energy of the gusts of wind at sea likely to be associated with microseisms, and also the pressures and variations of pressure at great depths on the bottom of the ocean likely to be associated with microseisms. It is hoped that, with further observations and consideration, more progress will be made, particularly with the air--sea energy transfer.

ERnest Tillotson

\section{BASIC RESEARCH ON ODOUR}

$\mathrm{T}$

O say that the sense of smell has been neglected as an object of study, because of the formidable experimental difficulties involved, particularly when examining the peripheral aspects of the sense, is of course to state a platitude. It would appear that the newer techniques of measuring minute quantities of substances might, however, prove rewarding when applied to the investigation of olfaction, and might be the means of stimulating renewed interest, especially in these neglected peripheral phases. It was possibly with such thoughts in mind that a conference was organized in April 1953, jointly by the New York Academy of Scionces and the American Society of Heating and Ventilating Engineers, for although practically every aspect of olfaction was then reviewed, greatest emphasis was placed on the peripheral phases. The edited versions of the papers read at the conference have now been published in the Annals of the New York Academy of Sciences*. No doubt because of the participation of the latter Society, papers on technological problems occupy a prominent place, more prominent than would probably be the case at a similar conference in Britain. The whole report, therefore, provides an up-to-date review, and a short guide to the literature, of both technical and laboratory work carried out recently on odours and olfaction.

A major object of any conference such as this is to throw up ideas, and a reasonable degree of speculation is not altogether undesirable. However, this can be overdone, and unfortunately several of the participants did not resist the temptation to explain how, on theoretical grounds, odours might be converted into nerve impulses. While reading some of the papers, one was reminded of Homer Smith's comment, made when he was reviewing the earlier concepts of renal function, that the history of science has been one of rival over-simplified theories, to which fault has been added that of insufficient experimental work. Parts of the report on this conference show that our grandfathers may have been no worse than ourselves.

The first part of the conference was conccrned with the problem of odour in industry ; the papers were, in fact, devoted to the practical problems of how to prevent food, tobacco, paper and the interiors of buildings from being contaminated by odoriferous

" "Basic Odour Research Correlation", by A. R. Behnke and 38 other specialists. Ann. N.Y. Acad. Sci., 58, Art. 2, 13-260 (1954). 3.50 dollars. substances. As there is so little basic knowledge available as to either the behaviour of the odoriferous substances, or the absorbent properties of the contaminated materials, the problems are attacked along empirical lines. Tho cause is traced, and if it cannot be removed then various odour neutralizers or modifiers are tried until the one which is most satisfactory in the circumstances has been found.

The next major section of the conference was entitled "Problems of Odour Research from the Viewpoint of the Scientist", with contributions from a physiologist, a psychologist, a physicist and a chemist. J. Greenspan outlines some of the newer physical methods for measuring concentrations as low as parts per million; but later in the conference, R. L. Kuehner reports that such methods are still not as satisfactory as would be desired, and he suggests the use of other methods which depend upon subjective threshold sensations, or upon chemical absorption of the odorant. L. M. Beidler, the physiologist, points out, too, that by analogy with the investigations on taste a method of measuring concentrations in the air, and of controlling such concentrations, would not necessarily enable the ultimate nature of the stimulus to be understood, for the nature of the receptor is so important. J. B. Sumner, at least, is aware that tho generally accepted view of odours being transmitted by volatile particles in the atmosphere is no more than a hypothesis, although undoubtedly a much more satisfactory one than others depending upon such an alternative as infra-red radiation.

The third section was concerned with the status of our present knowledge of the olfactory nervous system, and contained papers of rather uneven quality. There was in one paper a tabulated list of the functions of the turbinate bones; while another paper, which offered yet a further hypothesis of olfaction, based upon information theory this time, opened with a quotation followed by the comment, "In these words, Titus Lucretius Carus developed the first atomistic theory of olfaction in about 47 B.c. This year of 1953 marks the two-thousandth anniversary of this theory". On the other hand, K. H. Pribram and L. Kruger's review of the "Olfactory Brain" is particularly satisfying, and, at the risk of appearing invidious to the writers of other equally satisfactory articles, one may regard it as one of the most important papers of the conference and suggest that it is not to be overlooked. Their final conclusion justifies quoting: ". . . the 'olfactory brain', as defined, is not primarily olfactory, though parts of it serve olfactory functions. Nor is the current conception of a 'visceral brain' more tenable though viscero. autonomic functions are also served. It is clear that the formations in this portion of the brain, though they share several characteristics, are not, at this time, usefully thought of as a brain serving any one function. Since at least three distinct systems can be delineated, each might profitably be investigated before an attempt is made to define what functions they have in common". In the third section also, M. M. Mozell and C. Pfaffman contribute a review of the afferent neural processes, largely concerned naturally with Dr. E. D. Adrian's recont work, while V. G. Dethier has a most interesting paper on the physiology of olfaction in insects.

The rest of the conference was mainly devoted to techniques of measuring and identifying odours, with a consideration of some of the results obtained. 'This section might preferably have been placed earlier in. 
the conference. Most of the workers concerned with the practical problems of eliminating odours prefer to use subjective methods; but there is here also a full discussion of objective methods. It has been suggested that the olfactory mucosa is sensitive to concentrations as low as $10^{-13}$; if this is true, there is some considerable way to go, and much fundamental research is required, before adequate objective methods are available. But this is not to deprecate the very valuable and important work of the presentday pioneers.

H. SosNowICK

\section{AGE OF PARENT AND CHARACTER OF OFFSPRING}

$\mathrm{T}$ HE relations of an offspring to its parents are complex, and the ways in which a parent may influence the character of its offspring are many. This is especially true among the higher animals; but even when we turn to lower animals and to plants the parent-offspring relation, if simpler, is still far from simple. Though, as genetics has emphasized, the primary endowment is the complement of nuclear genes, an individual also receives from its parents its initial cytoplasm-mainly, but not necessarily exclusively, from its mother. The cytoplasm so received may contain particles of a genetic or semi-genetic nature (plasmagenes of various kinds), nutritional materials, protective substances and agents of disease (such as viruses). It is a complex endowment as yet by no means fully understood. Nor is the cytoplasm the only avenue of supply of food materials, protective substances and disease risks. The close association of offspring with parent during early life, especially in higher animals, plays its part in the nutrition, protection and disease relation of the offspring and, of course, also in its education.

Our understanding of these various links between the generations is not equal. Classically the geneticist has concerned himself primarily with the nucleus and the genes it carries, setting other aspects aside as secondary to the main problem. Initially this was an essential restriction, but of recent years especially the study of the nuclear genes has itself guided attention to the cytoplasm, to the immunological relations between mother and child, and to the effects of maternal nutrition; indeed, to the whole complexity of the parent-offspring relation. Thus as the genetical framework has become clear, the way has opened up to a co-operative attack on the broader problem by all the many interests which are concerned.

Both the complexity of the problem and the many types of approach which can be made to it are brought out by a recent symposium on "Parental Age and Characteristics of the Offspring" held by the New York Academy of Sciences*. Although in his introduction E. V. Cowdry chiefly emphasizes the effects of age of mother and father on longevity in the offspring, he recognizes in his summary that this provides only a superficial common denominator for the many phenomena described in the nineteen contributions to the symposium, and he stresses the need for a better understanding of the mechanisms which underlie the effects we observe. The observations reported cover a very wide field. Asexually produced progeny in plants are discussed alongside partheno* "Parental Age and Characteristics of the Offspring", edit. L. C.
Strong. Ann. N. Y. Acad. Sci., 57, 451-614 (1954). genetic rotifers and the sexual offspring of mammals. Longevity, germination characters of seeds, mutationrates, the expression of congenital malformation in men and mice, mongolism and the sex ratio in man, fibrocarcinoma and leukæmia in mice, the consequences of ageing gametes before fertilization, skeletal characters, spotting, the results of altering a mother's physiology during pregnancy, and seasonal influences, are all to be found among the subjects considered. As might be expected, the importance of the mother's age emerges clearly as opposed to that of the father; but there is, again not surprisingly, little pattern to be discerned as yet even at the level of description of the phenomena. Suggestions can be, and are freely, made as to the underlying causation. Hormonal changes, cytoplasmic entities, antibody levels, viral effects, biochemical adjustments, and alterations in crossingover with age, are all brought in, and the susceptibility of polygenically determined variants to non-nuclear effects is emphasized. But the value of this symposium lies in the problems it poses and the clues it offers rather than in the explanations it achieves or the mechanisms it exposes.

Two papers are especially interesting in their bearing on the nature of somatic ageing. The first, by A. I. Lansing, describes experiments with the rotifer Philodrina citrina, which, being parthenogenetic, is unlikely to show any significant amount of variation mediated by nuclear genes within its progenies. Separate lines were developed, differing in the age of the mother at the time of production of the eggs from which the next generation was raised. With eggs from adolescent mothers the longevity of the offspring tended to increase as the generations went by. The lines in which eggs were taken from middle-aged or senile mothers, on the other hand, showed a decline in longevity until they died out after five and three generations, respectively. The change is reversible by reversal of the selection. Furthermore, there is a close relation between growth and ageing, the ageing factor appearing in the eggs at the time when the mother ceases growth. Accelerated ageing accelerates growth but decreases maximal size, while retarded ageing retards growth and increases maximal size.

In the second paper E. Ashby and E. Wangerman describe experiments with clones of the water-plant Lemna minor. The fronds of this plant reproduce by asexual buds, the daughters so produced being successively smaller as the mother frond ages. It appears from extrapolation that the age of mother at which daughter size would be zero is the age of death. Thus the meristems grow old with the mother. As with the rotifers the process is cumulative and reversible, for first daughters are larger than their mothers where these mothers were themselves from old parents and therefore small.

The regularity and similarity of behaviour in the rotifer and the plant are striking. Though clearly not dependent on genetic segregation, the responses to selection resemble in several respects those which can be obtained with polygenic variation. Can they depend on a multi-particulate cytoplasmic system, related ultimately to the nucleus, but capable of change independently of the nuclear genes ? Though it is too early to do more than speculate, these papers suggest an experimental approach which might materially advance our understanding of the changes which go on during development and ageing.

K. MATHER 